\title{
Estudo Prospectivo das Tecnologias da Informação e Comunicação Aplicadas a Pessoas com Autismo
}

\author{
Prospective Study of Information and Communication Technologies \\ Applied to People with Autism
}

\begin{abstract}
Resumo
O autismo é um transtorno do desenvolvimento que normalmente costuma ser identificado na infância, persistindo na adolescência e na fase adulta. Pessoas com autismo são caracterizadas por déficits na comunicação, linguagem e comportamento, o que, invariavelmente, prejudica a sua interação social. Pesquisas em torno de Tecnologias da Informação e Comunicação (TIC), voltadas para o diagnóstico e o tratamento de pessoas com autismo, têm sido emergentes nos últimos anos. Dispositivos móveis, por exemplo, têm viabilizado diversas formas de acessibilidade e servido como ferramentas a indivíduos autistas com deficiências sensoriais, motoras, assim como aos que necessitam de comunicação aumentativa. Neste estudo, foram realizadas busca e análise em base de patentes e artigos científicos com o intuito de se obter um panorama das principais TICs que têm sido aplicadas a pessoas com autismo. Os resultados mostraram um crescimento substancial de trabalhos e tecnologias que podem proporcionar tratamentos alternativos para pessoas com autismo, totalizando 611 artigos científicos e 222 famílias de patentes.
\end{abstract}

Palavras-chave: Autismo. Tecnologia. TIC.

\begin{abstract}
Autism is a developmental disorder that is usually identified in childhood, persisting in adolescence and adulthood. People with autism are characterized by deficits in communication, language and behavior, which invariably impairs their social interaction. Research on information and communication technologies (ICT), focused on the diagnosis and treatment of people with autism, has been emerging in recent years. Mobile devices, for example, have enabled various forms of accessibility and served as tools for autistic individuals with sensory, motor disabilities, as well as those who need augmentative communication. In this study, a search and analysis was carried out on the basis of patents and scientific articles, in order to obtain an overview of the main ICTs that have been applied to people with autism. The results showed a substantial growth in works and technologies that can provide alternative treatments for people with autism, totaling 611 scientific articles and 222 patent families.
\end{abstract}

Keywords: Autism. Technology. ICT.

Área Tecnológica: Computação Aplicada à Saúde Humana. 


\section{Introdução}

O Transtorno do Espectro Autista (TEA), ou simplesmente o autismo, é entendido pela Organização Mundial da Saúde (OMS) como um grupo de condições caracterizadas por algum grau de comportamento social, comunicação e linguagem prejudicados, além de uma faixa estreita de interesses e atividades que são únicas para o indivíduo e realizadas repetidamente. Esse transtorno tende a surgir na infância, persistindo na adolescência até a fase adulta (WHO, 2019).

Ainda de acordo com a OMS, estima-se que uma a cada 160 pessoas no mundo tenha algum tipo de autismo. E, embora haja variação substancial nessa média de um estudo para o outro, o que se sabe é que o autismo tem aumentado em escala global, e as explicações para esse aumento são muitas, incluindo o crescimento da conscientização sobre o tema, a expansão dos critérios diagnósticos, melhores ferramentas de diagnóstico e o aprimoramento das informações reportadas (WHO, 2019).

De acordo com a Associação Americana de Psiquiatria (APA), há diferenças na gravidade e na combinação dos sintomas, de pessoa para pessoa, tais sintomas podem variar de leves a graves e mudar com o tempo. Além das duas categorias principais do transtorno do espectro autista, que são problemas na interação social e comunicação, e padrões restritos e repetitivos de interesse e atividades, algumas crianças e adultos com TEA podem desenvolver outros tipos de dificuldades psicológicas em algum momento de suas vidas, como ansiedade, TDAH, comportamentos perturbadores ou depressão. Essas dificuldades podem ser tratadas com terapia ou medicamentos, embora não existam medicamentos que tratem diretamente os principais problemas da TEA (APA, 2020).

Pesquisas têm sugerido que o TEA é um transtorno comportamental heterogêneo, com um forte componente genético, evidenciado por décadas de estudos. Esses avanços na genética têm possibilitado uma abordagem com forte potencial para diagnóstico e tratamento de medicina de precisão, bem como oportunidades de avançar na pesquisa científica básica sobre distúrbios do neurodesenvolvimento (ARNETT; TRINH; BERNIER, 2019). Por outro lado, muito ainda permanece desconhecido, isso devido à natureza frequente de variantes individuais, sendo que a penetrância para a TEA e os fenótipos relacionados a ela ainda não estão bem elaborados. Logo, muitos projetos de grande escala já foram iniciados para ajudar a compreender plenamente a complexidade subjacente da arquitetura genética do TEA (WOODBURY-SMITH; SCHERER, 2018).

A aplicação de tecnologias no ensino e no tratamento de pessoas com autismo tem crescido numa taxa impressionante (ODOM et al., 2014). De modo consequente, a mídia tem promovido, nos últimos anos, que o uso de dispositivos como smartphones, notebooks, tablets e outros, pode revolucionar o ensino acadêmico para estudantes autistas (KNIGHT; MCKSSICK; SAUNDERS, 2013). Os dispositivos móveis viabilizaram uma variedade de opções de acessibilidade que pode servir a pessoas com deficiências sensoriais e motoras, bem como a não leitores e àqueles com necessidades de comunicação aumentativa. Aliás, cada nova geração de tablets, smartphones e Personal Digital Assistent (PDA) tem oferecido recursos adicionais que podem ser adaptados para apoiar pessoas com autismo (GENTRY et al., 2014). 
$\mathrm{Na}$ atualidade, não apenas os tradicionais computadores têm auxiliado alunos com TEA nas escolas, mas especialmente os smartphones têm sido usados como apoio na aprendizagem e na conquista de independência do indivíduo no ambiente escolar, levando em consideração que os dispositivos móveis conectados à internet podem cumprir uma série de funções para o suporte organizacional, a comunicação, a interação social e o entretenimento (SILVA; CARVALHO; CAIADO, 2020). Pesquisas têm sugerido que jogos de computador, usados nesse contexto, como instrumento de intervenção no domínio da educação especial, podem gerar grandes êxitos no ensino de novas habilidades para crianças com TEA, tornando-as mais confiantes, menos ansiosas e mais ativamente engajadas (CHEN et al., 2019).

Todo o aparato de recursos e serviços que contribuem para proporcionar ou ampliar habilidades funcionais de pessoas com deficiência e, consequentemente, promover vida independente e inclusão é uma tecnologia assistiva. E esses recursos que podem variar de uma simples bengala a um complexo sistema computadorizado, incluindo brinquedos e roupas adaptadas, computadores, softwares e hardwares especiais, que contemplam questões de acessibilidade, dispositivos para adequação da postura sentada, recursos para mobilidade manual e elétrica, equipamentos de comunicação alternativa, chaves e acionadores especiais, aparelhos de escuta assistida, auxílios visuais, materiais protéticos e milhares de outros itens confeccionados ou disponíveis comercialmente (SARTORETTO; BERSCH, 2020).

Diversos fatores ajudam a explicar a afinidade de indivíduos autistas com os recursos computacionais. Primeiramente, pessoas autistas possuem dificuldades de filtrar informações sensoriais, $e$ as telas de computadores permitem que as informações sejam limitadas às informações relevantes, auxiliando o processo de filtragem. Em segundo lugar, indivíduos com autismo podem se confundir com imprevisibilidades, nuances sociais e mudanças repentinas do mundo físico, os computadores podem auxiliar nesse processo por serem muito mais previsíveis que os humanos, além do fato de que as interações computacionais podem ser repetidas indefinidamente, até que o usuário alcance o domínio. Outro fato é que computadores podem oferecer rotinas explícitas, ter expectativas claras e oferecer recompensas ou consequências para a resposta, incentivando o usuário ao envolvimento com tecnologias educacionais. Ademais, os dados coletados por sistemas computacionais também consistem numa valiosa fonte de informação para avaliar o progresso de usuários com autismo (SILVER; OKANE, 2001 apud KIENTZ et al., 2013).

Um dos campos em crescimento na pesquisa científica sobre tecnologias que possam ajudar no desenvolvimento de crianças com autismo é o da construção de robôs sociais. Estudos têm sugerido que esses robôs podem ajudar as crianças com autismo a desenvolverem habilidades sociais, desafiando a visão de que crianças autistas preferem tecnologias a outros tipos de atividades, engajamento com outros seres vivos ou interesse em relações humanas (RICHARDSON et al., 2018). Por outro lado, a interação criança-robô também tem sido usada como ferramenta de diagnóstico, ajudando na detecção precoce de sinais de TEA, pela observação comportamental naturalística e avaliações de comportamento. Isso tem proporcionado ganhos mais rápidos e significativos no diagnóstico e em intervenções terapêuticas, quando comparados a métodos clássicos (RAMÍREZ-DUQUE; FRIZERA-NETO; BASTOS, 2019). 
Assim, as inovações tecnológicas possuem um enorme potencial para auxiliar no diagnóstico precoce e melhorar os programas de intervenção, podendo ser categorizadas em rastreadores oculares, rastreadores de movimento, monitores de atividade eletrodérmica, sensores táteis, prosódia vocal e detectores de fala e dispositivos de avaliação da qualidade do sono (CABIBIHAN et al., 2016). O rápido desenvolvimento de tecnologias computacionais e da robótica na última década tem dado esperança em realizar um tratamento mais eficaz, consistente e econômico, além de dar precisão ao diagnóstico. Um dos principais benefícios do uso dessas tecnologias é que os estímulos produzidos a cada sessão do tratamento podem ser controlados, o que não só garante consistência nas diferentes sessões, mas, também, permite focar em um único fenômeno, pois pode ser difícil, até mesmo para profissionais treinados, realizar e entregar os estímulos de acordo com o plano de tratamento (LIU et al., 2017).

Entendendo a relevância dessa temática, este estudo se ateve a investigar as Tecnologias da Informação e Comunicação (TICs) utilizadas no tratamento de pessoas com autismo, por meio de base de patentes e de periódicos de artigos científicos. Logo, objetiva-se obter um panorama das principais TICs que têm sido emergentes nessa área, analisando suas classificações e campos de aplicações, identificando países em potencial na geração dessas tecnologias e correlacionando a produção tecnológica com a produção científica.

\section{Metodologia}

Para a realização deste trabalho foi feito um mapeamento prospectivo tendo como base os documentos de patentes e artigos científicos. Para o levantamento patentário, foi realizada uma pesquisa no sistema Questel Orbit, uma plataforma com grande abrangência de publicações, incluindo documentos de patentes de 87 escritórios nacionais e de seis escritórios regionais - EPO, WIPO, OAPI, ARIPO, EAPO e CGC - além de possuir ferramentas robustas que possibilitam diversas análises de informações contidas nos documentos de patente.

Para realizar o levantamento de publicações científicas, foi utilizada a base Scopus, o maior banco de dados de resumos e citações da literatura com revisão por pares, abrangendo revistas científicas, livros, processos de congressos e publicações do setor, incluindo ferramentas de análise e verificação da pesquisa.

As buscas nas referidas bases foram realizadas no mês de outubro de 2019, considerando o corte temporal de 2009 a 2019. Foram definidas strings de busca (Quadro 1) utilizando-se de operadores booleanos ("OR" e "AND") e truncadores (*) para que a pesquisa abranja variações de palavras que reflitam o objeto deste estudo. No caso da plataforma Scopus, a string foi aplicada nos campos título, resumo e palavras-chave. Para o sistema Orbit, a string foi inserida nos campos título e resumo. Em ambos os casos, as ferramentas disponíveis nas plataformas foram utilizadas para ajudar no refinamento dos dados. 
Quadro 1 - String de busca utilizada para a pesquisa de artigos científicos e patentes

\begin{tabular}{|c|c|} 
Scopus & QUESTEL-ORBIT \\
\hline TITLE-ABS-KEY ( ( ( system OR software OR program & ((system OR software OR program OR \\
OR device OR platform OR tool OR app*) AND & device OR platform OR tool OR app*) AND \\
autis*) ) AND DOCTYPE (ar) AND PUBYEAR $>$ & autis*)/TI/AB/IW AND APD $>=2009$ \\
2008 AND ( LIMIT-TO ( SUBJAREA, "COMP") &
\end{tabular}

Fonte: Elaborado pelos autores deste artigo (2019)

\section{Resultados e Discussão}

A investigação de patentes e artigos científicos retornou um total de 246 pedidos de depósito de patentes na base Questel Orbit e 611 artigos científicos publicados na base Scopus. Para encontrar resultados específicos para o objeto deste estudo, foi colocado o filtro de subárea "Ciência da Computação" na base Scopus e o filtro de domínio de tecnologia "Computação Tecnológica e Tecnologia Médica" no sistema Orbit. Patentes relacionadas a fármacos, material químico e alimentos foram excluídas dos resultados. A string utilizada nas duas bases e seus resultados são apresentados no Quadro 2.

Quadro 2 - Resultado da busca de artigos científicos e documentos de patentes

\begin{tabular}{|c|c|}
\hline $\begin{array}{c}\text { SCOPUS } \\
\text { SubÁREA: } \\
\text { CiÊNCIA dA CoMPUTAÇÃo }\end{array}$ & $\begin{array}{c}\text { ORBIT } \\
\text { Domínio da Tecnologia: } \\
\text { Computação Tecnológica E Tecnologia Médica }\end{array}$ \\
\hline Artigos publicados: 611 & Patentes depositadas: 222 \\
\hline
\end{tabular}

Fonte: Elaborado pelos autores deste artigo (2019)

O número de artigos científicos relacionados a TICs para o tratamento do autismo cresceu nos últimos 10 anos, tendo seu pico no ano de 2019, com 109 artigos publicados. Essa evolução pode ser observada na Figura 1, que traz o número de publicações científicas por ano de publicação. Também é possível notar certo crescimento no número de depósito de patentes relacionadas a TICs aplicadas ao autismo, sendo o ápice o ano de 2018, com 43 famílias de patentes, conforme também ilustra a Figura 1. Vale ressaltar que é possível que esse número cresça em 2019, tendo em vista os prazos do processo de depósito, já que em geral esses prazos impõem sigilo aos documentos até que ele seja publicado.

Figura 1 - Comparativo de distribuição de publicações de artigos científicos e famílias de patentes por ano
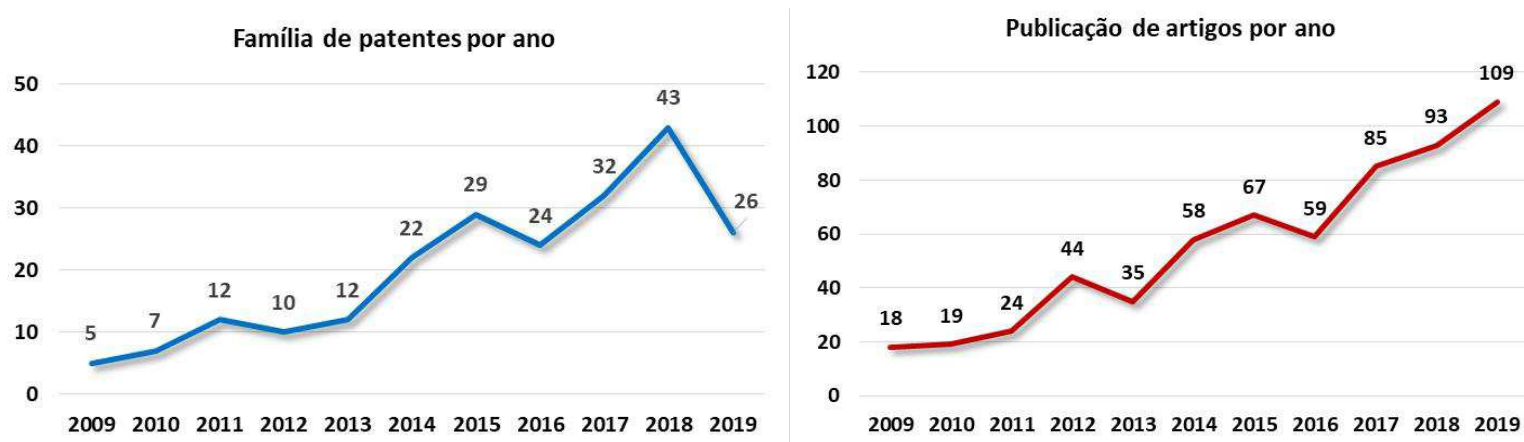

20092010201120122013201420152016201720182019

Fonte: Elaborada pelos autores deste artigo (2019) 
O aumento da produção de conhecimento científico e do desenvolvimento de novas tecnologias que auxiliem o tratamento e o aprendizado de pessoas com autismo pode ser explicado pela conscientização sobre o tema, que tem estado cada vez mais em evidência, principalmente nos últimos dez anos. Governos de diversos países têm estimulado políticas públicas que melhorem a vida de pessoas com autismo. Em geral, essas políticas incluem o diagnóstico precoce, a educação básica, a educação adaptada, o acesso ao mercado de trabalho, a assistência médica e os serviços de apoio domiciliares e comunitários (ASA, 2019).

Apesar do crescimento do número de depósitos de patentes nos últimos dez anos, nota-se, pelos dados da Figura 1, uma disparidade quando esse número é comparado ao crescimento da produção científica. Isso denota que os estudos e as pesquisas em torno de tecnologias para o autismo têm sido substanciais, embora tecnologias podem não estar sendo necessariamente protegidas de maneira legal. Para Cabibihan et al. (2016), em estudo de revisão de literatura sobre o estado da arte de tecnologias para detecção do autismo, muitas dessas tecnologias ainda não se encontram disponíveis para uso clínico e terapêutico por diversos motivos, como a ausência de avaliações rigorosas que atestem a viabilidade dessas tecnologias, especialmente em crianças, pois muitos estudos não passam da fase inicial devido à natureza diversificada dos sujeitos submetidos a testes. É imprescindível que evidências empíricas mostrem a repetibilidade, a robustez da tecnologia e demonstrem resultados comparáveis aos padrões de medição aceitos. Para Kientz et al. (2013), embora a condição do autista tenha sido conhecida desde a década de 1940, cada vez mais a sua complexidade tem sido compreendida, tendo ainda muito o que ser entendido sobre os desafios do autismo na vida cotidiana e, consequentemente, muito que pode ser feito para enfrentá-los.

Quanto às principais regiões, em termos de proteção de tecnologias e produção de conhecimento científico, algumas análises podem ser levantadas com base na Figura 2, que traz os dez principais países depositantes de patentes e produtores de conhecimento científico sobre o tema. É notória a forte predominância da China como principal país depositante de famílias de patentes, contabilizando 103 famílias de patentes, seguido dos Estados Unidos, apresentando importante destaque com 61 famílias de patentes. Os países contratantes do European Patent Office (EPO) somam, ao todo, 20 famílias de patentes. Vale destacar que o EPO agrega 38 países da Europa, e, por meio desse escritório, é possibilitado depositar um único pedido equivalente a pedidos de patentes nacionais, nos vários Estados contratantes da Convenção de Munique, sobre a Patente Europeia. Além do Japão, Canadá, Índia, Coreia do Sul, Austrália e Brasil, também é possível observar famílias de patentes depositadas via PCT - Tratado de Cooperação em Matéria de Patente (WO), em que, similarmente ao EPO, países de todo o mundo, que são signatários desse tratado, podem reivindicar proteção de tecnologias em vários países por meio de um único pedido de depósito.

De acordo com Clark, Zhou e Zheng (2019), a China investiu consideravelmente na última década em estudos epidemiológicos sobre o autismo, incluindo uma das pesquisas mais ambiciosas sobre a genética do transtorno, o que levou o país a considerá-lo uma prioridade, fornecendo maiores recursos às populações para garantir tratamentos acessíveis e opções educacionais apropriadas. 
Figura 2 - Gráficos dos 10 principais países por publicação de artigos e famílias de patentes
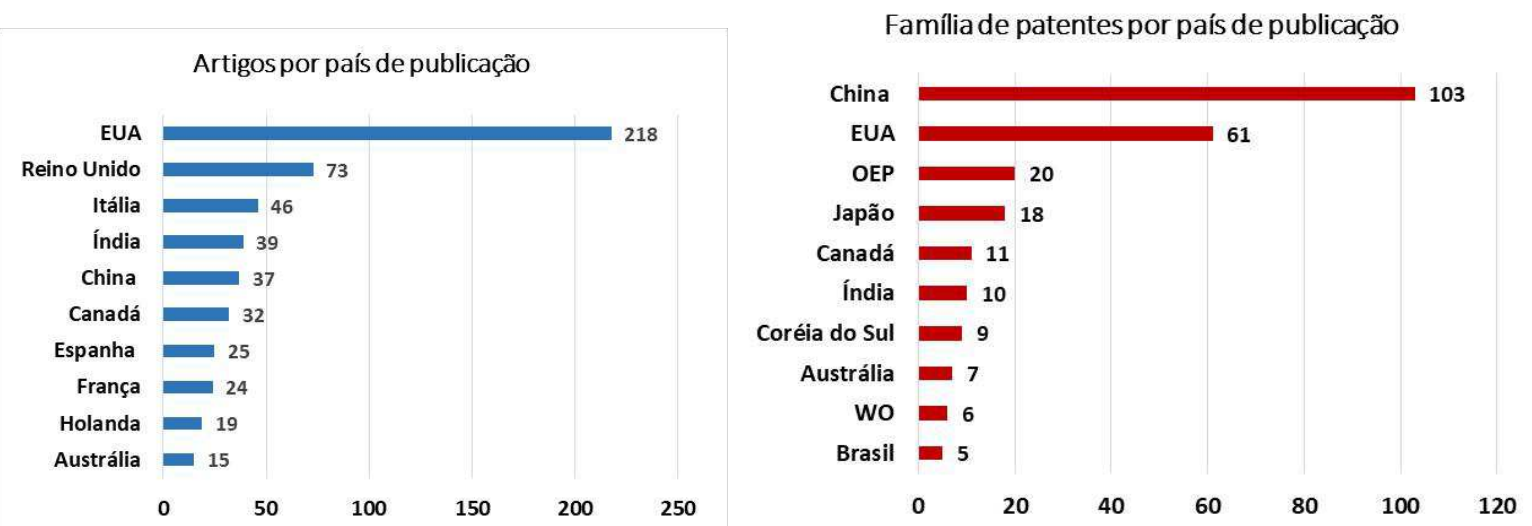

Fonte: Elaborada pelos autores deste artigo (2019)

Já em termos de produção de conhecimento científico, os Estados Unidos se sobressaem entre os dez principais países, com uma substancial produção científica de 218 artigos. Vale destacar que os esforços do governo americano em torno da problemática do autismo tiveram seu marco em 2006, quando a Lei de Combate ao Autismo foi assinada pelo então presidente George W. Bush, que previa apoio federal aprimorado à pesquisa e ao tratamento do autismo, criando um programa nacional de educação para os médicos e para a sociedade em geral sobre o autismo (THE WHITE HOUSE, 2006). Atualmente, a Academia Americana de Pediatria recomenda que toda criança seja submetida a uma triagem quanto a distúrbios do desenvolvimento que caracterizem o autismo antes dos três anos de idade (ACADEMIA AMERICANA DE PEDIATRIA, 2019).

É evidente, nesse resultado, a expressiva presença europeia entre esse grupo de países, tendo cinco (Reino Unido, Itália, Espanha, França e Holanda) dos dez países apresentados. A produção científica desses dez principais países representa cerca de $86,5 \%$ de toda a produção científica recuperada neste estudo. Pode-se notar que cinco países entre os principais depositantes de famílias de patentes também integram os dez maiores países em termos de publicação científica, sendo eles a China, os EUA, a Índia, o Canadá e a Austrália. Embora o número de pedidos de depósito de patentes seja expressivamente menor do que o número de publicações de artigos científicos.

A Figura 3 ilustra essa comparação entre publicação de artigos e pedidos de depósito de patentes, apresentando os cinco principais países (China, Canadá, EUA, Japão e Índia) além dos países que fazem parte da EPO. Nesse caso foi contabilizada a quantidade total de artigos científicos dos países signatários a esse acordo, é possível notar que, com exceção da China, que possui uma produção tecnológica bem acima da sua produção de conhecimento científico, a maioria dos países tem focado seus esforços nos meios científicos quanto à questão das TICs para o tratamento do autismo. No caso do Japão, apesar de os números em ambos os casos serem baixos, essa diferença entre artigos e patentes foi menor. 
Figura 3 - Quadro comparativo da produção de artigos versus família de patentes dos principais países

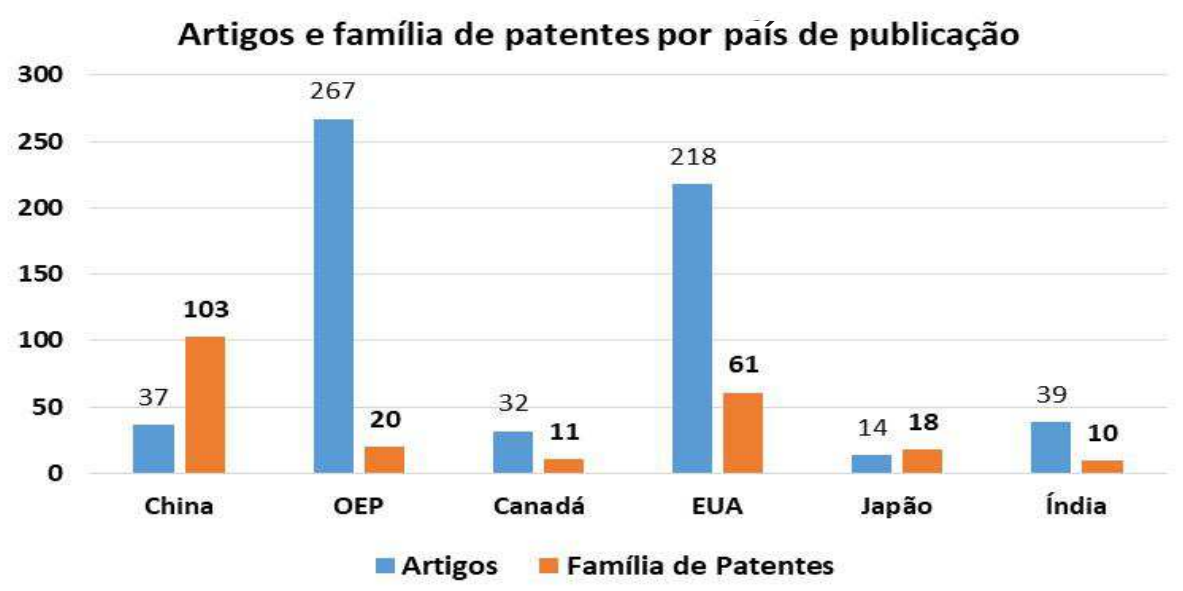

Fonte: Elaborada pelos autores deste artigo (2019)

Quando se compara as maiores instituições científicas e os maiores destinatários de depósito de patentes (Figura 3), é possível notar que a presença de universidades é considerável em ambos os casos. O que denota que o interesse acadêmico em cima da temática do autismo, como a busca de novas formas de diagnóstico e tratamento por meio do uso de tecnologias, tem sido exaustivamente aplicado. Embora o interesse mercadológico ainda possa ser embrionário devido às complexidades e as especificidades que os estudos acerca do autismo demandam.

Quanto à produção científica, destaca-se o Consiglio Nazionale dele Ricerche, o maior conselho de pesquisa científica da Itália com sede em Roma, com 19 produções científicas, seguido da Vanderbilt University, uma das 15 melhores universidades americanas da atualidade. Também se destacam nesse ranking, com a produção de oito artigos cada, a University of Heartordshire (Reino Unido), a University of Toronto (Canadá), o Centre National de la Recherche Scientifique (França), a University of Kansas Medical Center (EUA), e com a produção de sete artigos cada, a Stanford University (EUA), a University of Missouri-Columbia (EUA), a Nanyang Technological University (Singapura) e a Harvard Medical School (EUA).

Figura 4 - Ranking das principais instituições e/ou empresas produtoras de artigos científicos e tecnologias

Artigoscientíficos por afiliação



Famílias de patentes por Destinatário

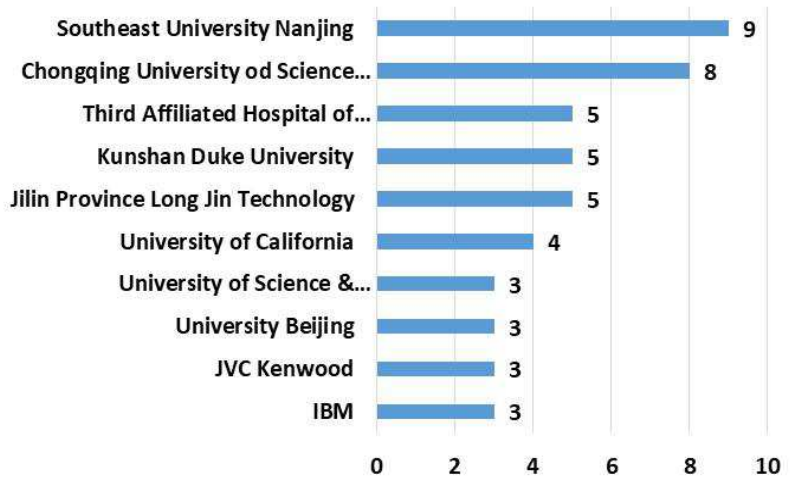

Fonte: Elaborada pelos autores deste artigo (2019) 
Como já destacado, este estudo observou que universidades possuem protagonismo não apenas na produção científica, mas também no desenvolvimento de TICs para o tratamento do autismo. De acordo com a Figura 4, no topo do ranking dos principais requerentes de patentes, são apresentadas duas universidades de grande renome na China: Southeast University Nanjing e Chongqing University of Science and Technology, com nove e oito famílias de patentes, respectivamente. Outras quatro universidades chinesas também figuram esse ranking, contabilizando cinco famílias de patentes cada, o Third Affiliated Hospital of Zhongshan University, Kunshan Duke University, e com três famílias de patentes cada, a University of Science \& Technology Beijing e a University Beijing. A University of California aparece com quatro patentes, sendo a única universidade fora da China nesse cenário.

Entre esses dez maiores depositantes, três empresas também são apontadas, sendo elas a Jilin Province Long Jin Technology (5 famílias de patentes), empresa chinesa especializada em dispositivos de aprendizado de alta tecnologia voltados para crianças; a multinacional japonesa JVC Kenwood (3 famílias de patentes), especializada em tecnologias de som e vídeo, principalmente para automóveis; e a gigante americana IBM (3 famílias de patentes), atuante no mercado da tecnologia da informação que tem se destacado por suas tecnologias avançadas, desde computadores de grande porte até nanotecnologia.

Essa análise mostra que a produção tecnológica e científica de TICs voltadas para o autismo está bastante concentrada nas instituições acadêmicas, visto o considerável número de universidades nos rankings de produção de artigos e depósito de patentes. Por se tratar de uma problemática complexa e que continua, diariamente, a ser investigada por estudiosos $e$ especialistas, é verossímel que as universidades sejam as grandes protagonistas na busca por soluções científicas e tecnológicas para auxiliar autistas em terapias e diagnósticos. Ademais, devido à própria heterogeneidade dos transtornos observados em pessoas com autismo, o que individualiza a solução e implica mais estudos investigativos, é provável que parte dessas tecnologias demandem mais tempo para, de fato, se tornarem um produto de mercado.

A Classificação Internacional de Patentes (CIP) é utilizada por escritórios de patentes de mais de 100 países para classificar seus documentos. Além de auxiliar na busca e na recuperação de documentos de patentes, o código CIP permite organizá-los, facilitando o acesso às informações tecnológicas e legais, serve de base para investigar o estado da técnica de determinados campos da tecnologia e é usado como base para a elaboração de estatísticas em propriedade industrial em diversas áreas.

Neste estudo, as principais classificações CIP estão relacionadas às classes A61B-005/16 (25,66\%) e A61M-021/00 (20,35\%), como pode ser observado na Figura 6. O grupo A61B abrange instrumentos, implementos e processos para fins de diagnose, cirurgia ou identificação de pessoas, inclusive obstetrícia, instrumentos para remover calos, instrumentos para vacinação, datiloscopia e exames psicofísico. Já o grupo A61M engloba dispositivos para introduizr matérias no corpo ou depositá-las sobre ele, dispositivos para fazer circular matérias no corpo ou para dele as retirar e dispositivos para por fim ao sono ou à letargia. A Figura 6 ilustra as dez principais classes e subclasses das famílias de patentes investigadas neste estudo. O Quadro 3 apresenta a descrição de cada uma delas. 
Figura 6 - Famílias de patentes por principais códigos CIP

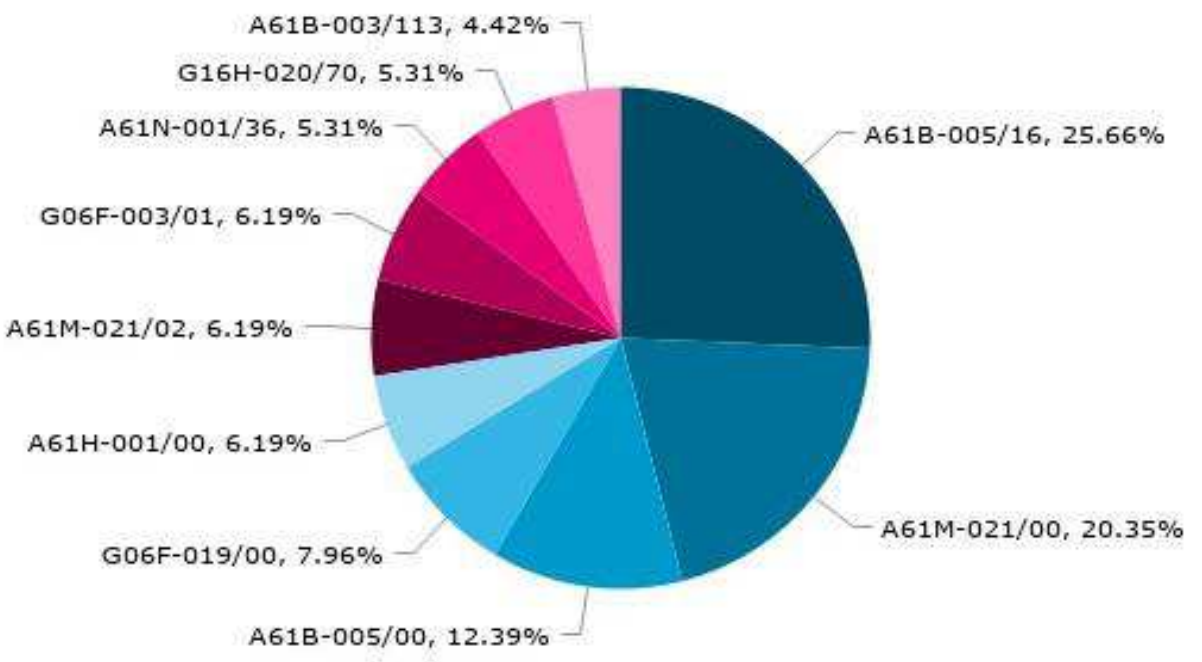

Fonte: Questel Orbit (2019)

Quadro 3 - Principais códigos CIP e suas respectivas descrições

\begin{tabular}{|c|c|}
\hline CÓDIGO CIP & DESCRIÇÃO \\
\hline A61B-005/16 & Dispositivos para exames psicotécnicos (usando dispositivos educacionais ou de ensino). \\
\hline A61M-021/00 & $\begin{array}{l}\text { Outros dispositivos ou métodos para produzir mudança no estado de consciência; } \\
\text { Dispositivos para produzir ou interromper o sono por meios mecânicos ópticos } \\
\text { ou acústicos, por exemplo, por hipnose (camas para favorecer o sono). }\end{array}$ \\
\hline A61B-005/00 & $\begin{array}{l}\text { Medição com finalidades de diagnóstico (diagnóstico por radiação; } \\
\text { diagnóstico por ondas de ultrassom, sônicas ou infrassônicas). }\end{array}$ \\
\hline G06F-019/00 & $\begin{array}{l}\text { Química teórica computacional, i.e. ICT especialmente adaptada para aspectos teóricos } \\
\text { da química quântica, mecânica molecular, dinâmica molecular ou semelhantes. }\end{array}$ \\
\hline A61H-001/00 & $\begin{array}{l}\text { Aparelhos para exercícios passivos; Aparelhos vibratórios; Dispositivos para } \\
\text { quiroprática, por exemplo, dispositivos para impacto do corpo, dispositivos } \\
\text { externos para ligeira extensão ou alinhamento de ossos quebrados. }\end{array}$ \\
\hline A61M-021/02 & $\begin{array}{l}\text { Dispositivos ou métodos para induzir ao sono ou relaxamento, por exemplo, por estímulo } \\
\text { direto ao nervo, hipnose, analgesia (para massagem; eletroterapia, por exemplo, pela } \\
\text { aplicação de correntes elétricas alternadas ou intermitentes para produzir anestesia). }\end{array}$ \\
\hline G06F-003/01 & $\begin{array}{l}\text { Disposições de entrada ou disposições combinadas de entrada } \\
\text { e saída para interação entre usuário e computador. }\end{array}$ \\
\hline A61N-001/36 & Eletroterapia; Seus circuitos para estimulação, por exemplo, marcapassos cardíacos. \\
\hline
\end{tabular}

Fonte: Elaborado pelos autores deste artigo (2019)

Outros grupos de CIP também se destacam nesse ranking, como o grupo $\mathrm{A} 61 \mathrm{H}$, mais especificamente o subgrupo 001/00, que se refere a aparelhos para exercícios passivos, aparelhos vibratórios e dispositivos para quiroprática, que podem indicar tecnologias que auxiliam em processos fisioterapêuticos e outros tratamentos ligados ao sistema musculoesquelético. Tecnologias relacionadas à eletroterapia (grupo A61N-001/36), prática amplamente usada na 
fisioterapia, inclusive, também aparecem nesse ranking. O uso dessas tecnologias em sessões fisioterápicas pode ser fundamental para o indivíduo autista, devido às dificuldades motoras que muitos desenvolvem. Tecnogias ligadas a práticas de relaxamento e indução de sono (grupo A61M-021/02) também se destacam nesse levantamento, levando em consideração que problemas ligados à ansiedade são pertinentes em indivíduos com TEA. Já o grupo G06F-003, que está relacionado a disposições de entrada ou disposições combinadas de entrada e saída para interação entre usuário e computador, indica os atuais sistemas computacionais desenvolvidos sob a forma de diferentes dispositivos.

A Figura 7 apresenta o quantitativo de patentes por domínio tecnológico, mais especificamente os dez principais domínios. Como pode ser visto, a maior parte das patentes se refere ao domínio da tecnologia médica, precisamente tecnologias de diagnóstico, odontologia, implantes, terapias e farmacêuticos, contabilizando um total de 180 famílias de patentes. Já os sistemas computacionais somam 70 famílias de patentes do que foi apurado neste estudo. Tecnologias relacionadas a controladores, à educação e à criptografia representam 20 famílias de patentes. Em número menor também é possível notar outros importantes domínios tecnológicos dentro da temática do autismo, como tecnologias relacionadas a games (12 patentes), ao reconhecimento de voz (12 patentes), à computação de métodos de negócios ( 5 patentes), à robótica (5 patentes), à óptica (4 patentes), à comunicação digital (4 patentes) e ao audiovisual (3 patentes).

Figura 7 - Patentes por domínio tecnológico

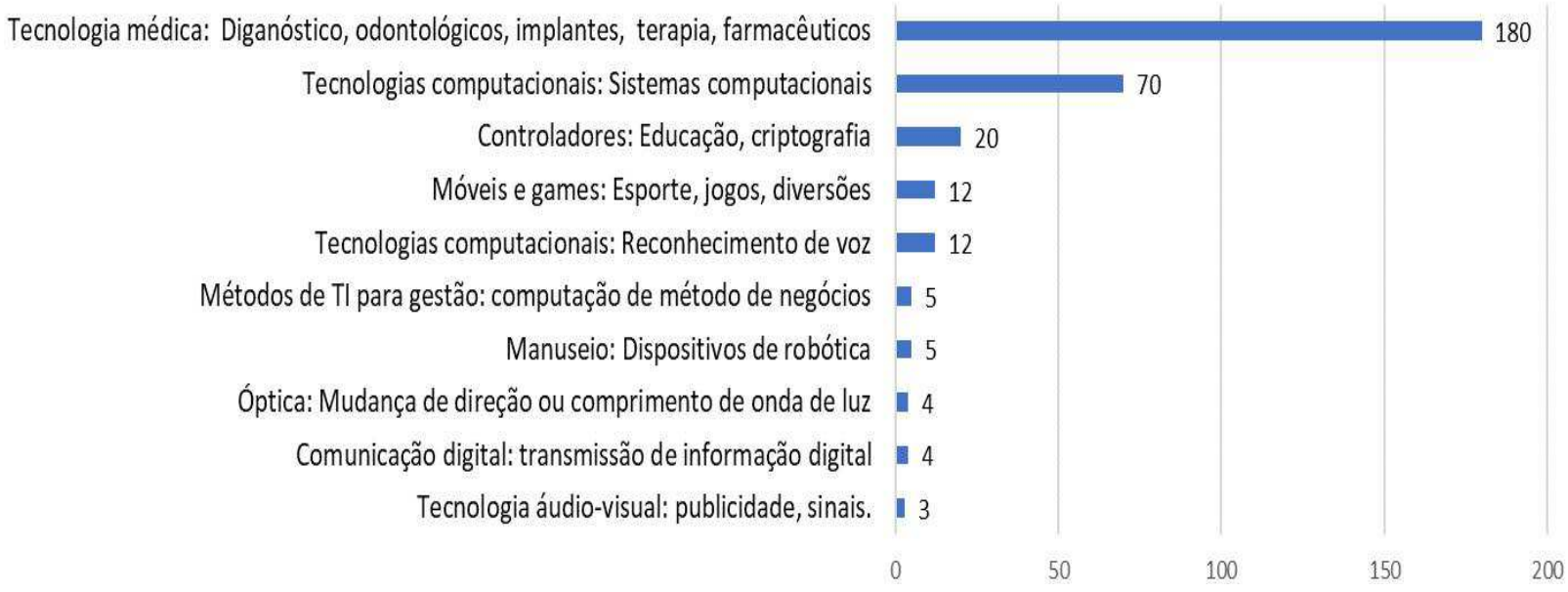

Fonte: Questel Orbit (2019)

Vale destacar que, da mesma forma que a classificação por CIP, uma patente pode ser categorizada em mais de um domínio tecnológico.

Na Figura 8 é possível observar as principais palavras-chave encontradas nos artigos levantados neste estudo. Pode-se notar que termos das TICs, como realidade virtual, robótica, aprendizado de máquina, mineração de dados, interação homem-robô, reconhecimento de emoções, tecnologias assistivas, computação pervasiva, entre outros, associados ao autismo têm sido extensivamente pesquisados e publicados em artigos científicos. Muitas dessas pesquisas centradas especialmente em crianças se dedicam a investigar alternativas de tratamento e de diagnóstico, entendendo os principais sintomas do autismo, que são essencialmente manifestados na comunicação e na interação do indivíduo com o seu meio. 
Figura 8 - Nuvem de palavras-chave identificadas nos artigos encontrados



Fonte: Elaborada pelos autores deste artigo (2019)

\section{Considerações Finais}

Este artigo apresentou um estudo de prospecção científica e tecnológica de TICs relacionadas ao tratamento e ao diagnóstico do autismo por meio de levantamento científico e patentário sobre a temática.

A prospecção mostrou que o desenvolvimento de estudos científicos e de tecnologias relacionadas ao autismo tem sido uma forte tendência nos últimos dez anos, dado o expressivo crescimento de artigos científicos e de depósitos de patentes sobre essa temática. Tendo a China e os EUA como os países mais fortemente interessados no desenvolvimento dessas soluções, tanto por meio de estudos científicos como no investimento patentário.

O interesse acadêmico sobre esse tema também foi evidenciado, não apenas na produção de artigos científicos como também em depósitos de patentes, o que atesta que as pesquisas de novas formas de tecnologias para o tratamento e o diagnóstico do autismo têm sido exaustivamente realizadas, embora o interesse mercadológico ainda possa ser embrionário. Patentes de tecnologias médicas para fins de diagnósticos e terapêuticos e sistemas computacionais foram as classificações que mais se destacaram nesta análise. Mas também houve expressivo número de estudos científicos que tratam de temas como robótica, realidade virtual e aprendizado de máquina.

Ademais, o trabalho mostrou que o desenvolvimento de estudos e de tecnologias de soluções para a problemática autista tem sido diversificado, embora, considerando a própria complexidade do TEA, esse ainda seja um campo aberto ao desenvolvimento de inovações e estudos.

\section{Perspectivas Futuras}

Com o aumento das políticas públicas nos países para a conscientização do autismo em todo o mundo, é provável que os estudos científicos e o desenvolvimento de inovações tecnológicas sejam ainda mais impulsionados, nos próximos anos, em torno dessa temática. Apesar da expressividade de artigos e de patentes apresentada neste estudo, ainda existem muitas lacunas 
e gargalos dentro da complexidade do autismo que demandarão novas soluções tecnológicas e densos estudos. Sugere-se aqui, como estudos futuros, a realização de prospecções ainda mais específicas dentro desse tema, por exemplo, o monitoramento de tecnologias de dispositivos móveis para educação de crianças com autismo e uma prospecção de tecnologias classificadas pelo código CIP $(\mathrm{G} 16 \mathrm{H})$, que classifica as TICs especialmente adaptadas para fins específicos.

\section{Referências}

ACADEMIA AMERICANA DE PEDIATRIA. How Pediatricians Screen for Autism. 2019.

Disponível em: https://www.healthychildren.org/English/health-issues/conditions/Autism/Pages/HowDoctors-Screen-for-Autism.aspx. Acesso em: 11 jan. 2021.

APA - AMERICAN PSYCHIATRY ASSOCIATION. What is Autism Spectrum Disorder? [2020]. Disponível em: https://www.psychiatry.org/patients-families/autism/what-is-autism-spectrum-disorder. Acesso em: 5 jan. 2021.

ARNETT, Anne B.; TRINH, Sandy; BERNIER, A. Raphael. The state of research on the genetics of autism spectrum disorder: methodological, clinical and conceptual progress. Current Opinion In Psychology, [s.l.], v. 27, p. 1-5, jun. 2019. Elsevier BV. Disponível em: https://pubmed.ncbi.nlm.nih. gov/30059871/. Acesso em: 11 jan. 2021.

ASA - AUTISM SOCIETY OF AMERICA. (USA). Public Policy. [2020]. Disponível em: https:/www. autism-society.org/public-policy/. Acesso em: 11 jan. 2021.

CABIBIHAN, J. et al. Sensing Technologies for Autism Spectrum Disorder Screening and Intervention. Sensor (Basel), [s.l.], v. 17, ed. 46, 2016.

CLARK, Elaine; ZHOU, Zheng; DU, Lin. Autism in China: progress and challenges in addressing the needs of children and families. International Journal of School \& Educational Psychology, [s.l.], v. 7, n. 2, p. 135-146, 3 abr. 2019. Informa UK Limited.

CHEN, Jingying et al. A pilot study on evaluating children with autism spectrum disorder using computer games. Computers in Human Behavior, [s.l.], v. 90, p. 204-214, 2019.

GENTRY. T. et al. Reducing the Need for Personal Supports Among Workers with Autism Using an iPod Touch as an Assistive Technology: Delayed Randomized Control Trial. Journal of Autism and Developmental Disorders, Nova York. v. 45, n. 3, p. 669-684, 2014.

KIENTZ, J. et al. Interactive Technologies for Autism. Morgan \& Claypool Publishers, University of Toronto, 2013.

KNIGHT, V.; MCKSSICK, B. R.; SAUNDERS, A. A Review of Technology-Based Interventions to Teach Academic Skills to Students with Autism Spectrum Disorder. Journal of Autism and Developmental Disorders, Nova York, v. 43, n. 11, p. 2.628-2.648, 2013.

LIU, Xiongyi et al. Technology-Facilitated Diagnosis and Treatment of Individuals with Autism Spectrum Disorder: an engineering perspective. Applied Sciences, [s.l.], v. 7, n. 10, p. 1.051, 13 out. 2017. MDPI AG. Disponível em: https:/www.mdpi.com/2076-3417/7/10/1051/htm. Acesso em: 11 jan. 2021. 
ODOM, S. L. et al. Technology-Aided Interventions and Instruction for Adolescents with Autism Spectrum Disorder. Journal of Autism and Developmental Disorders, Nova York. v. 45, n. 12, p. 3.805-3.819, 2014.

QUESTEL ORBIT [Base de dados - Internet]. Questel Orbit Inc. 2019. Disponível em: https:// www.orbit.com/. Acesso em: 31 out. 2019.

RAMÍREZ-DUQUE, Andrés A.; FRIZERA-NETO, Anselmo; BASTOS, Teodiano Freire. Robot-Assisted Autism Spectrum Disorder Diagnostic Based on Artificial Reasoning. Journal of Intelligent \& Robotic Systems, [s.l.], v. 96, n. 2, p. 267-281, 29 mar. 2019. Springer Science and Business Media LLC. Disponível em: https://ink.springer.com/article/10.1007/s10846-018-00975-y\#article-info. Acesso em: 11 jan. 2021.

RICHARDSON, Kathleen et al. Robot Enhanced Therapy for Children with Autism (DREAM): a social model of autism. Ieee Technology And Society Magazine, [s.l.], v. 37, n. 1, p. 30-39, mar. 2018. Institute of Electrical and Electronics Engineers (IEEE). Disponível em: https://ieeexplore.ieee. org/abstract/document/8307127. Acesso em: 11 jan. 2021.

SARTORETTO, Mara Lúcia; BERSCH, Rita. O que é Tecnologia Assistiva? [2020]. Disponível em: https://www.assistiva.com.br/tassistiva.html. Acesso em: 11 jan. 2021.

SILVA, Josiane Almeida da; CARVALHO, Michele Elias de; CAIADO, Roberta Varginha Ramos; BARROS, Isabela Barbosa Rêgo. As tecnologias digitais da informação e comunicação como mediadoras na alfabetização de pessoas com transtorno do espectro do autismo: uma revisão sistemática da literatura. Texto Livre: Linguagem e Tecnologia, Minas Gerais, v. 13, n. 1, p. 4564, 11 jul. 2020. Disponível em: https://periodicos.ufmg.br/index.php/textolivre/article/view/24069. Acesso em: 11 jan. 2021.

THE WHITE HOUSE. Fact Sheet: Combating Autism Act of 2006. Disponível em: https:// georgewbush-whitehouse.archives.gov/news/releases/2006/12/20061219-3.html. Acesso em: 14 out. 2019.

WOODBURY-SMITH, Marc; SCHERER, Stephen W. Progress in the genetics of autism spectrum disorder. Developmental Medicine \& Child Neurology, [s.l.], v. 60, n. 5, p. 445-451, 25 mar. 2018. Wiley. Disponível em: https://pubmed.ncbi.nlm.nih.gov/29574884/. Acesso em: 11 jan. 2021.

WHO - WORLD HEALTH ORGANIZATION. Autism spectrum disorders. 2019. Disponível em: https://www.who.int/news-room/fact-sheets/detail/autism-spectrum-disorders. Acesso em: 11 jan. 2021.

\section{Sobre os Autores}

\section{Felipe Roberto Eloi Moura}

E-mail: felipemoura.al@gmail.com

ORCID: http://orcid.org/0000-0002-7509-1942

Mestre em Propriedade Intelectual e Transferência de Tecnologia para a Inovação pela Universidade Federal de Alagoas.

Endereço profissional: Rua Ariosvaldo Pereira Cintra, n. 152, Gruta de Lourdes, Maceió, AL. CEP: 57052-580. 


\section{Josealdo Tonholo}

E-mail: tonholo@gmail.com

ORCID: http://orcid.org/0000-0002-4015-9564

Doutor em Físico-Química pela Universidade de São Paulo.

Endereço profissional: Universidade Federal de Alagoas, Instituto de Química e Biotecnologia, Departamento de Química, CCEN/UFAL, Tabuleiro do Martins, Maceió, AL. CEP: 57072970.

\section{Alana Jéssica Vilela Messias}

E-mail: alanamessias@gmail.com

ORCID: http://orcid.org/0000-0003-4760-3434

Especialista em Informática pela Faculdade Internacional Signorelli.

Endereço profissional: Universidade Federal de Alagoas, Reitoria, AC Cidade Universitária, Maceió, AL. CEP: 57072970.

\section{Bruna Pinto de Cerqueira Pedrosa de Oliveira}

E-mail: brunapcpo@gmail.com ORCID: http://orcid.org/0000-0001-9796-9181

Mestre em Propriedade Intelectual e Transferência de Tecnologia para a Inovação pela Universidade Federal de Alagoas.

Endereço profissional: Rua Vista do Atlântico, n. 246, Jacarecica, Maceió, AL. CEP: 57042-532. 